\title{
On the Relation Between GRB Classes and X-ray Flashes
}

\author{
Z. Bagoly, P. Veres, I. Horváth, A. Mészáros, L. G. Balázs
}

\begin{abstract}
Gamma-ray bursts are usually classified into either short-duration or long-duration bursts. Going beyond the short-long classification scheme, it has been shown on statistical grounds that a third, intermediate population is needed in this classification scheme. We are looking for physical properties which discriminate the intermediate duration bursts from the other two classes. As the intermediate group is the softest, we argue that we have related them with X-ray flashes among the GRBs. We give a new, probabilistic definition for this class of events.
\end{abstract}

Keywords: gamma rays, bursts, observations.

\section{Introduction}

To discern the physical properties of GRBs as a whole, we need to understand the number of physically different underlying classes of the phenomenon. With the launch of the Swift satellite [1], a new perspective has opened up in the study of gamma-ray bursts and their afterglows. The intermediate GRB population in the studies [2-5] so far has always been the softest among the groups, meaning that intermediate GRBs emit the bulk of their energy in the low-energy gamma-rays.

Here we report on a significant difference in the peak-flux distribution between the intermediate and the short populations, and between the intermediate and long populations. We identify a third population using a multi-component model and we show that this group has a significant overlap with Xray flashes. The First Swift BAT Catalog [6] was augmented with bursts up to August 7, 2009. After excluding the outliers and bursts without measured parameters, our sample has a total of 408 GRBs. To obtain the spectral parameters we fitted the spectra integrated for the duration of the burst with a power law model and a power law model with an exponential cutoff. The most widely used duration measure is $T_{90}$, which is defined as the period between the $5 \%$ and $95 \%$ of the incoming counts. To find the fluences $\left(S_{E_{\min }, E_{\max }}\right)$ we integrated the model spectrum in the usual Swift energy bands with $15-25-50-100-150 \mathrm{keV}$ as their boundaries. We define the hardness ratio $\left(H_{i j}\right.$, where $i$ and $j$ mark the two energy intervals) as the ratio of the fluences in different channels for a given burst.

\section{Classification}

There are many indications that the phenomenon which we observe as gamma-ray bursts has more than one underlying population. The goal is to identify classes which are physically different. By using $T_{90}$ and $H_{32}$ we include a basic temporal and spectral characteristic of the bursts. We carry out three types of classifications: model-based multivariate classification, k-means clustering and hierarchical clustering. We use the algorithms implemented in the $\mathrm{R}$ software $^{1}$.

Studies show that for example the distribution of the logarithm of the duration can be adequately described by a superposition of three Gaussians [2]. Here we find the model parameters using the Expectation-Maximization (EM) maximum likelihood method. We use the Bayesian Information Criterion (BIC), introduced by $[7,8]$, to find the most probable model (including the number of components) and the parameters of this model. For $k$ components (bivariate Gaussians) the number of free parameters is $6 k-1$, since the sum of the weights is 1 .

We have applied this classification scheme on our sample, and found that the model with three components gives the best fit for the data in the BIC sense, where the shape of the bivariate Gaussians is the same $\left(\sigma_{\log _{10} T_{90}, i}=\sigma_{\log _{10} T_{90}, j}\right.$ and $\sigma_{\log _{10} H_{32}, i}=$ $\sigma_{\log _{10} H_{32}, j}$ for $i, j=$ \{short, long, intermediate $\}$ ) for each group, only their weights are different with no correlation, the best model has a value of $B I C=$ -262.14 . The clustering method of this model shows that a three bivariate component model is the most preferred. Two components models have the best $B I C \sim-276$ and for models with four components the best $B I C \sim-274$, both are clearly below the maximum. The best-fit model has 10 free parameters and has three bivariate Gaussian components. We assign class memberships probabilities using the ratio of the fitted bivariate models at the burst location on the duration-hardness plane. The model has the following three components with equal stan-

\footnotetext{
${ }^{1}$ http://cran.r-project.org
} 
Table 1: Bivariate model parameters for the best-fitted (EEI) model. The standard deviations in the direction of the coordinate axes and the correlation coefficients are constrained by the model

\begin{tabular}{cccccccc}
\hline Groups & $p_{l}$ & $\lg T_{90 C}$ & $\lg H_{32 C}$ & $\sigma_{\lg T_{90}}$ & $\sigma_{\lg H_{32}}$ & $r$ & $N_{l}$ \\
\hline \hline short & 0.08 & -0.331 & 0.247 & 0.509 & 0.090 & 0 & 31 \\
interm. & 0.12 & 1.136 & -0.116 & 0.509 & 0.090 & 0 & 46 \\
long & 0.80 & 1.699 & 0.114 & 0.509 & 0.090 & 0 & 331 \\
\hline
\end{tabular}

dard deviations in both directions and with no correlation $(r=0)$ (see also Table 1$)$ :

1. The first component is the known short class of GRBs (shortest duration and hardest spectra). The average duration is $0.47 \mathrm{~s}$ and the average hardness ratio is 1.77 . It has 31 members, and the weight of this model component is 0.08 .

2. The second, most numerous model component is the long class, also identified in many previous studies. It has an average duration of $50.0 \mathrm{~s}$ and an average hardness of 1.30 . It has $331 \mathrm{mem}-$ bers and the weight of the model component is 0.80 .

3. The third and softest class is intermediate in duration. It has overlapping regions with previous definitions of the intermediate class [4]. The average duration is $13.7 \mathrm{~s}$, and the average hardness of this class is 0.77 . It has 46 members and the weight of the model component is 0.12 .

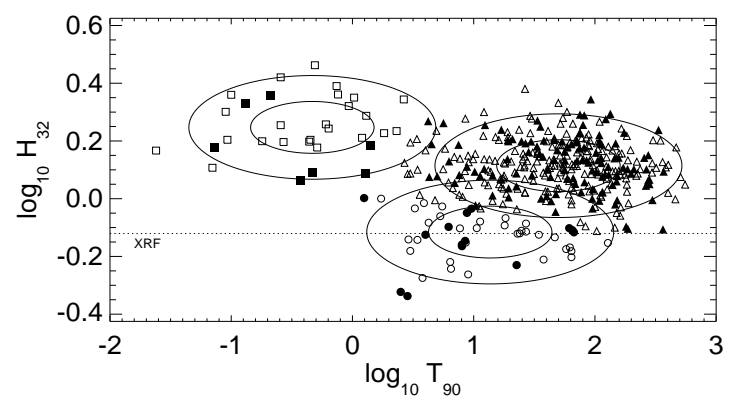

Fig. 1: GRB populations on the duration-hardness plane. Triangles mark the long class, squares mark the short class and circles mark the intermediate class. Filled symbols mark bursts with measured redshifts. One and two sigma ellipses are superimposed on the figure to illustrate the model components found as described in the text. The dashed line indicates the definition of X-ray flashes (XRFs) given by [9]

All components have the same standard deviation in both directions. This means the shape of the Gaussian is the same for the three groups (though obviously their weight is different). Models with non-zero correlation coefficients between the two variables are not favored in the BIC sense, contrary to the models with $r=0$.
Using both model-based and non-parametric kmeans and hierarchical clustering methods we have experimented by using $T_{50}$ instead of $T_{90}$, by using different hardness ratios (e.g., $H_{42}=\frac{S_{100-150}}{S_{25-50}}$, $H_{432}=\frac{S_{100-150}}{S_{25-50}+S_{50-100}}$ etc.). The classification remained essentially the same.

\section{Discussion}

Our analysis on the Swift GRBs supports the earlier results that there are three distinct groups of bursts. Again, besides the long and the short population, the intermediate duration class appears to be the softest.

The intermediate bursts' peak-flux are systematically lower than the long ones, while their redshift range is either lower or similar. We thus conclude that the intermediate class is intrinsically dimmer. If the intermediate population is part of the long population, the lower peak-flux requires a physical explanation. The observational properties show that intermediate bursts are the softest among the three groups, meaning that their emission is concentrated to low-energy bands.

As the intermediate population is the softest, it is worth searching for a link with the similar and softer phenomenon compared to classical gammaray bursts, the X-ray flashes (XRFs) (for a review, see [11]). [9] gives a working definition for X-ray flashes (XRF) and X-ray rich GRBs (XRR) for Swift using the fluence ratio. The $S_{23}$ fluence ratio is the reciprocal of the hardness $\left(H_{32}=\left(S_{23}\right)^{-1}\right)$. Current understanding of XRFs indicate that they are related to long bursts and they form a continuous distribution in the peak energy $\left(E_{\text {peak }}\right)$ of the $\nu F_{\nu}$ spectrum [9].

According to the fuzzy classification model we do not get a definite membership for a given burst, rather a probability that a burst belongs to a group. To identify the intermediate population (and tentatively the X-ray flashes), we use the indicator function:

$$
\begin{aligned}
& I_{\text {Interm. }}\left(\log _{10} T_{90}, \log _{10} H_{32}\right)= \\
& \sum_{\text {interm. }} \times P\left(\log _{10} T_{90}, \log _{10} H_{32} \mid " \text { Interm." }\right) \\
& \sum_{1} \times P\left(\log _{10} T_{90}, \log _{10} H_{32} \mid l\right)
\end{aligned}
$$


The values of the parameters in this equation should be taken from Table 1 . The joint distribution function of the fitted model can be seen in gray in Figure 2 and the probability contours of the third population are drawn in black with probability level contours shown.

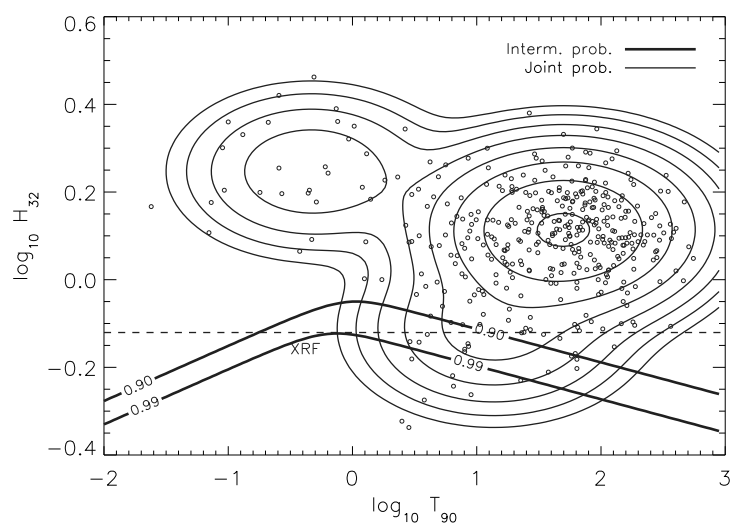

Fig. 2: Contour plot of the Swift's duration-hardness distribution based on the EEI model with three components. Points show individual bursts. The dashed line shows the region belonging to the XRF population, and the indicator function of the intermediate group is shown by solid lines. One can observe the strong coincidence between the XRFs and the intermediate group

[9] define XRFs as events with hardness ratio $H_{32}<0.76$. The limit is found using a pseudo-burst with spectral parameters: $\alpha=-1, \beta=-2.5$ and $E_{\text {peak }}=100 \mathrm{keV}$ for a Band spectrum [10]. Based on this definition we identify 24 bursts from our 408 burst sample. The average of these bursts' probabilities, (i.e. the XRF belongs to the intermediate group) is $95 \%$. This high value allows us to conclude that all XRFs belong to the intermediate group defined by the EEI model with high probability. We propose that the members of the third component are probably X-ray flashes. Therefore, using the model based classification method we can give a probabilistic definition for the X-ray flashes based on the durationhardness distribution. This definition defines 22 additional bursts that belong to the intermediate population and hence to the XRFs.

All the X-ray flashes are in the region where the third component has the highest probability, but not all third component bursts can be unambiguously classified as X-ray flashes according to the [9] criterion. In other words the third component in the EEI model contains all the X-ray flashes and some additional, very soft bursts.

The mechanism behind the X-ray flashes is still not clear. There are various scenarios that could produce these phenomena (e.g. dirty fireballs, inefficient internal shocks, structured jets with off-axis viewing angle, etc., for a review of the models see [12]). A more precise experimental definition of XRFs can result in more stringent constraints on the models.

\section{Acknowledgement}

This work was supported by OTKA grant K077795, by OTKA/NKTH A08-77719 and A08-77815 grants (Z.B.), by GAČR grant No. P209/10/0734 (A.M.), by the Research Program MSM0021620860 of the Ministry of Education of the Czech Republic (A.M.) and by a Bolyai Scholarship (I.H.). We thank Peter Mészáros, Gábor Tusnády, Lídia Rejtő and Jakub Rípa for valuable comments.

\section{References}

[1] Gehrels, N., et. al: ApJ, 611, 1005-1020.

[2] Horváth, I., et. al: $A \& A, 447,23-30$.

[3] Huja, D., Mészáros, A., Řípa, J.: $A \& A$, 504, 67.

[4] Horváth, I., et. al: $A \mathscr{G} A, \mathbf{7 1 3}, 552$.

[5] Rípa, J., et. al: $A \mathscr{E} A, \mathbf{4 9 8}, 399$.

[6] Sakamoto, T., et. al: ApJ Suppl., 175, 179-190.

[7] Schwarz, G.: Annals of Statistics, 6, 2, 461-464.

[8] Liddle, A. R.: MNRAS, 377, L74-L78.

[9] Sakamoto, T., et. al: ApJ, 679, 570-586.

[10] Band, D., et. al: ApJ, 413, 281-292.

[11] Hullinger, D.: Early afterglow evolution of x-ray flashes observed by Swift, $\mathrm{PhD}$ thesis, University of Maryland, College Park, 2006.

[12] Zhang, B.: Chinese Journal of Astronomy and Astrophysics, 7, 1-50.

\section{Z. Bagoly}

Department of Physics of Complex Systems

Eötvös University

1518 Budapest, Pf. 32, Hungary

Department of Physics

Bolyai Military University

1581 Budapest, POB 15, Hungary

P. Veres

Department of Physics of Complex Systems

Eötvös University

1518 Budapest, Pf. 32, Hungary

Department of Physics

Bolyai Military University

1581 Budapest, POB 15, Hungary

Konkoly Observatory

1505 Budapest, POB 67, Hungary 
I. Horváth

Department of Physics

Bolyai Military University

1581 Budapest, POB 15, Hungary

L. G. Balázs

Konkoly Observatory

1505 Budapest, POB 67, Hungary

\section{A. Mészáros}

Astronomical Institute

Faculty of Mathematics and Physics

Charles University

V Holešovičkách 2, 18000 Prague 8, Czech Republic 